\title{
MODELO DE ESTIMATIVA DE TEMPERATURAS MÁXIMAS E MÍNIMAS MENSAIS PARA A REGIÃo DO MÉDIO PARANAPANEMA, SP
}

\author{
Angelica Prela Pantano ${ }^{1}$, Ludmila Bardin ${ }^{2}$
}

\begin{abstract}
RESUMO
A temperatura do ar é um dos elementos mais importantes e deve ser levado em consideração em qualquer estudo relacionado ao ser vivo. Este trabalho foi desenvolvido para a região do Médio Paranapanema, com o objetivo de estimar as temperaturas médias do ar, devido a ausência de postos meteorológicos na região. Foram utilizados dados de nove municípios devido ao fato de apenas esses terem uma série mais longa de dados coletados. Modelos de estimativa de temperatura do ar, com base em coordenadas geográficas foram utilizadas com a finalidade de estimar as temperaturas médias mensais das máximas e das mínimas para a região. Equações de regressão múltipla em função da altitude, da latitude e da longitude, foram obtidos, os coeficientes de determinação variaram de 0,76 a 0,89 para as temperaturas máximas e de 0,39 a 0,96 para as mínimas, sendo que os menores coeficientes foram encontrados nos meses mais frios, encontrou-se alta relação entre altitude e médias anuais de temperatura, evidenciando que o modelo pode ser utilizado para estimativa de temperaturas médias anuais em todos os municípios da região do Médio Paranapanema.
\end{abstract}

Palavras-chave: equação de Regressão, modelagem, SIG.

\section{ABSTRACT \\ MODEL AND ESTIMATE OF MONTHLY MAXIMUM AND MINIMUM TEMPERATURE IN CENTRAL PARANAPANEMA REGION, SP}

The air temperature is one of the most important factors to be considered in any study related to a living being. Due to lack of meteorological stations, this study was done for the central Paranapanema region, to estimate average air temperatures. The data from nine cities that had long series of collected data were used. Models were used to estimate monthly mean maximum and minimum air temperatures of the region, based on geographical coordinates. Multiple regression equations were obtained depending on altitude, latitude and longitude. The coefficients of determination ranged from 0.76 to 0.89 for maximum and 0.39 to 0.96 for the minimum temperature. For the colder months the coefficients were lower. There was high correlation between altitude and mean annual temperatures, showing that the model can be used to estimate annual mean temperatures in all cities in the central Paranapanema.

Keywords: regression equation, modeling, SIG.

\section{Recebido para publicação em 09/02/2012. Aprovado em 16/04/2012.}

1- Eng. Agr. Dra ${ }^{\mathrm{a}}$, Pesquisadora, IAC/APTA/SAA, Campinas, SP, e-mail: angelica@iac.sp.gov.br

2- Eng. Amb., Doutoranda, IAC, Campinas, SP, e-mail: ludmila_bardim@yahoo.com.br 


\section{INTRODUÇÃO}

A temperatura do ar é uma das variáveis atmosféricas mais importantes e deve ser levada em consideração em qualquer estudo relacionado ao meio ambiente. Esse interesse ocorre devido ao fato desse elemento exercer influência direta sobre todos os seres vivos. Neste sentido, inúmeros estudos já foram e vem sendo realizados com o objetivo de estimar a temperatura do ar de determinado local. Cada espécie de ser vivo, seja vegetal ou animal, desenvolve melhor suas funções e desenvolvimento fisiológico quando submetida às condições específicas de temperatura. Essas condições são denominadas temperatura basal inferior, ótima e basal superior, e seu conhecimento é fundamental para determinação das unidades térmicas exigidas por plantas e animais para o seu desenvolvimento em sub-período ou ciclo de crescimento e desenvolvimento (PRELA; RIBEIRO, 2002).

Nos Estados Unidos da América, Fernandez (1992) desenvolveu um método empírico para simular temperaturas médias mensais e anuais do ar para localidades com altitude abaixo de $1500 \mathrm{~m}$. Fuming (1988) estimou a temperatura do ar para períodos curtos (10 dias), em função da latitude e da altitude.

No Brasil, métodos para estimar temperaturas vêm sendo desenvolvidos e utilizados há algum tempo, e são relevantes quando se trata de localidades onde há escassez desses dados (BURIOL et al., 1973, ALFONSI, 1974; FEITOSA et al., 1979, 1980a e 1980b; SANDANIELO, 1987; CAVALCANTI et al., 1994; LIMA; RIBEIRO, 1998; ABREU et al. 2011).

No estado de São Paulo, foram estimadas as temperaturas para as regiões litorâneas e verificado que não é recomendado, para estimativa, para o Vale do Ribeira, em função de altitude e latitude local (PEDRO JR. et al., 1991). Bardin et al. (2010) estimaram temperaturas médias mensais das máximas e das mínimas para a região conhecida como Circuito das Frutas, no estado de São Paulo. Esses encontraram coeficientes de determinação $\left(\mathrm{R}^{2}\right)$ menos elevados, nos meses de inverno, o que também foi detectado anteriormente por Pedro JR. et al. (1991).

No estado do Paraná, Galvani et al. (2000), também desenvolveram modelos de estimativa do ar para a região de Maringá, utilizando como base as coordenadas geográficas locais e a temperatura do ar medida no abrigo meteorológico (1,5 $\mathrm{m}$ de altura) e ainda a nebulosidade, estimaram a temperatura do ar em curto prazo.

De acordo com Medeiros et al. (2005), o ajuste das equações de regressão para estimativa de temperaturas é uma alternativa viável para ampliar a base de dados climáticos, por meio de mapas de temperatura, podendo ser utilizado como subsídios no planejamento agrícola, como também a técnica de regressão linear múltipla e krigagem ordinária para modelagem de temperatura adotada por Lado et al. (2007).

A região do médio Paranapanema situa-se ao sudoeste do estado de São Paulo e faz divisa com o Paraná. Possui cerca de 40 municípios e uma área considerada faixa transicional dos climas zonais, caracterizado por maior irregularidade interanual das precipitações. A região é caracterizada ainda pela agropecuária, sendo boa parte do seu território cultivado com grãos (soja, milho e trigo) e cultivos semiperenes como banana, mandioca, café, cana-deaçúcar e pastagens. Essa região possuí 25 estações meteorológicas, instaladas, na grande maioria nos últimos anos, e o curto período de dados observados é insuficiente para as análises propostas, uma vez que, segundo Prela-Pantano et al. (2009), são necessários um mínimo de sete anos de dados para caracterizar as condições de temperatura dessa região.

Face ao exposto, o objetivo desse trabalho é desenvolver e testar equações de estimativa de temperatura para a região do Médio Paranapanema, em função das coordenadas geográficas (altitude, latitude e longitude) e fornecer subsídios para estimar temperaturas médias de locais onde não existem postos meteorológicos e registro dessas informações.

\section{MATERIAL E MÉTODOS}

A área de estudo engloba municípios da região sudoeste do estado de São Paulo, que compõem o chamado Vale do Médio Paranapanema (Figura 1). A região compreende 32 municípios, dos quais 10 possuem postos com coleta de dados meteorológicos a mais de nove anos. Foram utilizados valores de temperaturas máxima e mínima coletados diariamente que foram agrupados para obtenção das médias mensais de cada local e posteriormente 
calculados a média da região. Foram utilizadas as séries de dados de temperatura de 8 municípios, os quais possuem séries completas e com mais de 9 anos. As séries variaram de nove a quinze anos e foram obtidas junto ao banco de dados do Centro Integrado de Informações Agrometeorológicas (CIIAGRO) do Instituto Agronômico de Campinas, que mantém estações meteorológicas instaladas na região. As coordenadas geográficas das localidades analisadas estão descritas no Quadro 1. Os dados foram submetidos à análise estatística para obtenção das equações de regressão múltipla para posterior obtenção das temperaturas estimadas.

As temperaturas médias mensais máximas e mínimas foram ajustadas pelo método dos mínimos quadrados, obtendo-se as equações de regressão múltipla. $\mathrm{y}=\mathrm{a}+\mathrm{bx}+\mathrm{cx}_{1}+\mathrm{dx}_{2}$

em que

$\mathrm{y}=$ é a temperatura média mensal máxima ou mínima $\left({ }^{\circ} \mathrm{C}\right)$;

$a, b, c$ e d = são os coeficientes da equação de regressão; $\mathrm{x}$ - é a altitude (metros);

$\mathrm{x}_{1}=$ é a latitude (minutos); e

$\mathrm{x}_{2}=$ é a longitude (minutos).

De posse das equações de regressão para estimativa das temperaturas foi calculada a variação espacial em ambiente SIG-ILWIS e, elaborado os mapas das temperaturas máximas e mínimas da região de estudo. A verificação da correlação entre a altitude e a temperatura das estações foi realizada pela planilha de cálculos do Excel.

Quadro 1. Municípios das estações meteorológicas, coordenadas geográficas e período avaliado na região do Médio Paranapanema, SP

\begin{tabular}{lcccc}
\hline \multicolumn{1}{c}{ Município } & Altitude $(\mathrm{m})$ & Latitude $(\mathrm{min})$ & Longitude $(\mathrm{min})$ & Período \\
\hline Assis & 563 & 1360 & 3025 & 1991 a 2007 \\
Ipaussu & 574 & 1333 & 2857 & 1999 a 2007 \\
Manduri & 700 & 1362 & 2717 & 1992 a 2007 \\
Maracaí & 420 & 1357 & 3040 & 1998 a 2007 \\
Palmital & 501 & 1367 & 3013 & 1993 a 2007 \\
Paraguaçu Paulista & 489 & 1363 & 2721 & 1998 a 2007 \\
Pedrinhas Paulista & 330 & 1369 & 3048 & 1993 a 2007 \\
Tarumã & 441 & 1365 & 3035 & 1992 a 2007 \\
\hline
\end{tabular}

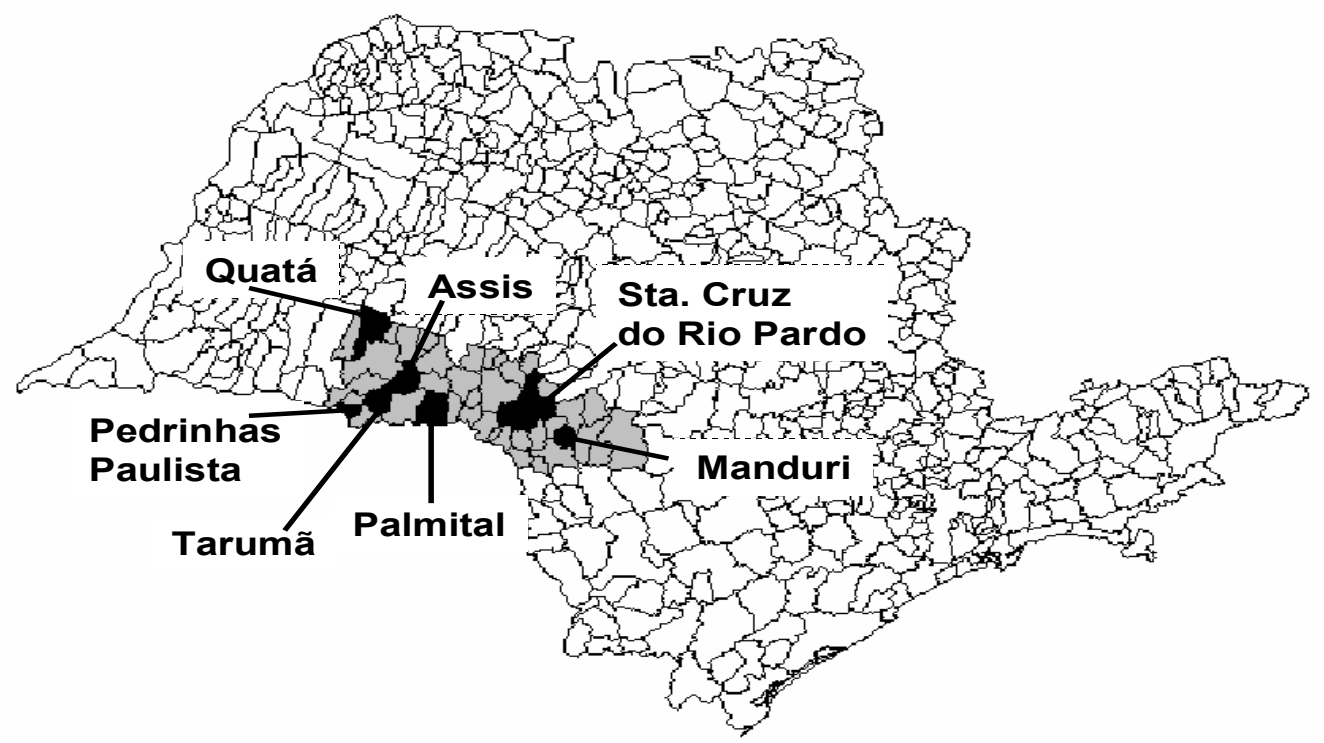

(Fonte: PRELA-PANTANO, et al., 2009).

Figura 1. Representação do Estado de São Paulo, identificando a região de estudo, Médio Paranapanema. 


\section{RESULTADOS E DISCUSSÕES}

Foram encontrados valores de coeficiente de determinação $\left(\mathrm{R}^{2}\right)$ elevados para estimativa de temperaturas máximas, variando de 0,76 a 0,89 , valores estes considerados satisfatórios. Em relação às temperaturas mínimas, foram encontrados coeficientes que podem ser divididos em dois grupos distintos. Os valores de $\mathrm{R}^{2}$ foram menores nos meses mais frios (abril a agosto), variando de 0,39 em junho e 0,61 em abril. Nos demais meses foram encontrados valores acima de 0,75 (Quadro 2). Em meses mais frios as equações de estimativas das médias das temperaturas mínimas são menos precisas, resultados estes que corroboram com os encontrados anteriormente por Pedro Jr. et al. (1991) e Bardin et al. (2010).

Quando correlacionadas às temperaturas observadas com as estimadas foram encontrados valores de coeficiente de correlação $\left(\mathrm{R}^{2}\right)$ variando de 0,1529 (junho) a 0,9389 (janeiro), para as temperaturas médias das máximas. Sendo que as correlações menores foram observadas nos meses mais frios. Para as temperaturas mínimas, os valores de correlação variaram de 0,3835 (junho) a 0,8109 (setembro) (Quadro 3).
Analisando-se os valores de $\mathrm{R}^{2}$ apresentados na tabela 5, pode-se afirmar que o método de estimativas de temperatura média mensal aplicado é menos eficiente quando se estima a média das temperaturas em meses mais frios. Nesses meses, foram observados os menores valores de coeficiente de correlação, tanto para as médias das temperaturas máximas quanto para as médias das mínimas mensais, o que também foi observado em outras regiões como o Vale do Ribeira (PEDRO JR et al.,1991) e na região conhecida como Circuito das Frutas (BARDIN, et al., 2010).

Observou-se que Pedrinhas Paulista é a localidade com temperatura média máxima anual mais elevada, $30,5^{\circ} \mathrm{C}$ e, Mandurí a localidade com menor temperatura média máxima anual dessa região, $\operatorname{com} 27,9^{\circ} \mathrm{C}$.

Em relação às temperaturas estimadas das médias das mínimas mensais Mandurí é a localidade mais fria, sendo que a temperatura mínima média anual está em torno de $15,1^{\circ} \mathrm{C}$, e Pedrinhas Paulista a menos fria, com temperatura mínima anual de $16,8{ }^{\circ} \mathrm{C}$. Com base nas temperaturas estimadas, pode-se considerar que Pedrinhas Paulista é localidade mais quente dessa região e Mandurí a mais fria.

Quadro 2. Valores de coeficientes das equações de regressão múltipla, encontrados para estimativa das médias mensais de temperatura máxima e mínima em função da altitude, latitude e longitude, para a região do Médio Paranapanema

\begin{tabular}{|c|c|c|c|c|c|c|c|c|c|c|}
\hline \multirow{2}{*}{ Mês } & \multicolumn{5}{|c|}{$\begin{array}{c}\operatorname{Tmax}=a+b(\text { alt }-m)+c(\text { lat-min })+d \\
\text { (long-min) }\end{array}$} & \multicolumn{5}{|c|}{$\begin{array}{c}\operatorname{Tmin}=a+b(\text { alt }-\mathrm{m})+\mathrm{c}(\text { lat }-\min )+\mathrm{d}(\text { long }- \\
\min )\end{array}$} \\
\hline & $\mathrm{a}$ & $\mathrm{b}$ & $\mathrm{c}$ & $\mathrm{d}$ & $\mathrm{R}^{2}$ & $\mathrm{a}$ & $\mathrm{b}$ & $\mathrm{c}$ & $\mathrm{d}$ & $\mathrm{R}^{2}$ \\
\hline Jan & 17,13 & $-0,0084$ & 0,0176 & $-0,0020$ & 0,89 & $-21,59$ & $-0,0040$ & 0,03436 & $-0,0011$ & 0,96 \\
\hline Fev & 39,70 & $-0,0075$ & $-0,0007$ & $-0,0014$ & 0,85 & $-16,22$ & $-0,0043$ & 0,03167 & $-0,0018$ & 0,89 \\
\hline Mar & 26,80 & $-0,0086$ & 0,0083 & $-0,0008$ & 0,86 & $-6,92$ & $-0,0050$ & 0,02304 & $-0,0009$ & 0,89 \\
\hline Abr & 45,49 & $-0,0098$ & $-0,0033$ & $-0,0021$ & 0,83 & 8,37 & $-0,0043$ & 0,00933 & $-0,0007$ & 0,61 \\
\hline Mai & 47,93 & $-0,0093$ & $-0,0054$ & $-0,0034$ & 0,82 & 10,35 & $-0,0043$ & 0,0055 & $-0,0010$ & 0,52 \\
\hline Jun & 57,63 & $-0,0092$ & $-0,0113$ & $-0,0039$ & 0,76 & 38,92 & $-0,0049$ & $-0,0139$ & $-0,0019$ & 0,39 \\
\hline Jul & 10,06 & $-0,0048$ & 0,0097 & 0,0016 & 0,81 & 7,65 & $-0,0041$ & 0,0079 & $-0,0018$ & 0,41 \\
\hline Ago & 35,16 & $-0,0088$ & $-0,0015$ & $-0,0002$ & 0,88 & 6,62 & $-0,0044$ & 0,00653 & $-0,0004$ & 0,52 \\
\hline Set & 18,42 & $-0,0099$ & 0,0117 & $-0,0003$ & 0,86 & $-15,97$ & $-0,0042$ & 0,02562 & $-0,0009$ & 0,75 \\
\hline Out & 46,70 & $-0,0086$ & $-0,0113$ & 0,0012 & 0,85 & $-34,67$ & $-0,0046$ & 0,04268 & $-0,0013$ & 0,81 \\
\hline Nov & 30,42 & $-0,0082$ & 0,0019 & 0,0006 & 0,88 & $-34,31$ & $-0,0058$ & 0,04476 & $-0,0021$ & 0,96 \\
\hline Dez & 28,18 & $-0,0082$ & 0,0098 & $-0,0022$ & 0,89 & $-44,24$ & $-0,0036$ & 0,04794 & $-0,00001$ & 0,93 \\
\hline $\begin{array}{l}\text { Média } \\
\text { Anual }\end{array}$ & 33,64 & -0 , & 0,0021 & $-0,0011$ & 0,85 & $-8,50$ & $-0,0045$ & 0,02212 & $-0,0012$ & 0,73 \\
\hline
\end{tabular}


Entre as médias das temperaturas mínimas observadas e das estimadas foi encontrada uma variação de $-1,5$ a $1,5^{\circ} \mathrm{C}$. Já nas médias das máximas mensais a variação encontrada foi de $-1,2$ a $1,0^{\circ} \mathrm{C}$. Observouse freqüência maior nas variações menores entre $-0,2$ e $0,5^{\circ} \mathrm{C}$. Comparando-se os valores de temperatura observados com os estimados pela equação, cerca de $80 \%$ encontram-se nesse intervalo (Figura 2).

A baixa correlação em meses mais frios pode estar associada ao fato da região estar localizada numa zona de transição (MONTEIRO, 1973) e exposta a frentes frias e massas de ar vindas do Estado do Paraná, e que são reforçadas e mais visíveis em épocas mais frias, ocasionando maior variação nas temperaturas.

Esse método foi aplicado para os demais municípios situados na região do Médio
Paranapanema e foi encontrada alta correlação entre a altitude $\mathrm{e}$ a as temperaturas médias anuais, em torno de $95 \%$. Quando foi estimada as temperaturas médias anuais em relação à altitude, observou-se que existe uma tendência de ocorrência de menor temperatura de acordo com o aumento da altitude (Figura 3).

Nas Figuras 4 e 5 está representada a região de estudo e as variações de temperaturas. As temperaturas mais elevadas foram observadas na região de divisa com o Estado do Paraná, margeando o Rio Paranapanema, principalmente nos municípios de Cruzália e Florínea (Figura 4). Em relação às temperaturas mais baixas, estas foram observadas nas localidades com maiores altitudes, como Echaporã, Quatá, Mandurí e Avaré (Figura 5), o que corrobora com estimativas representadas na Figura 4.

Quadro 3. Valores de coeficientes das equações de correlação $\left(\mathrm{R}^{2}\right)$ encontrados entre temperaturas observadas e temperaturas estimadas das médias mensais de temperatura máxima e mínima para a região do Médio Paranapanema $(\mathrm{y}=a \mathrm{x}+b)$

\begin{tabular}{lcccccc}
\hline \multirow{2}{*}{ Mês } & \multicolumn{3}{c}{ Temperatura Máxima } & \multicolumn{3}{c}{ Temperatura Mínima } \\
\cline { 2 - 7 } & $\mathrm{a}$ & $\mathrm{b}$ & $\mathrm{R}^{2}$ & $\mathrm{a}$ & $\mathrm{b}$ & $\mathrm{R}^{2}$ \\
\hline Jan & 0,9389 & 1,214 & 0,9389 & 1,0075 & 0,0325 & 0,7503 \\
Fev & 0,7988 & 3,9317 & 0,7988 & 0,7147 & 8,8443 & 0,7172 \\
Mar & 0,7976 & 3,8681 & 0,7976 & 0,8297 & 5,4902 & 0,7697 \\
Abr & 0,3769 & 10,547 & 0,3769 & 0,6579 & 10,229 & 0,6565 \\
Mai & 0,2787 & 9,0615 & 0,2787 & 0,6132 & 9,9713 & 0,6239 \\
Jun & 0,1529 & 10,176 & 0,1529 & 0,4333 & 14,58 & 0,3835 \\
Jul & 0,1741 & 9,2187 & 0,1741 & 0,6781 & 8,3894 & 0,5697 \\
Ago & 0,2714 & 8,8072 & 0,2714 & 0,7665 & 6,5713 & 0,7670 \\
Set & 0,5760 & 6,0469 & 0,5760 & 0,8570 & 4,2682 & 0,8109 \\
Out & 0,6711 & 5,6370 & 0,6711 & 0,7020 & 8,9349 & 0,6597 \\
Nov & 0,9234 & 1,3561 & 0,9234 & 0,7938 & 6,3643 & 0,7838 \\
Dez & 0,8560 & 2,7472 & 0,8560 & 0,7251 & 8,6864 & 0,6141 \\
\hline
\end{tabular}

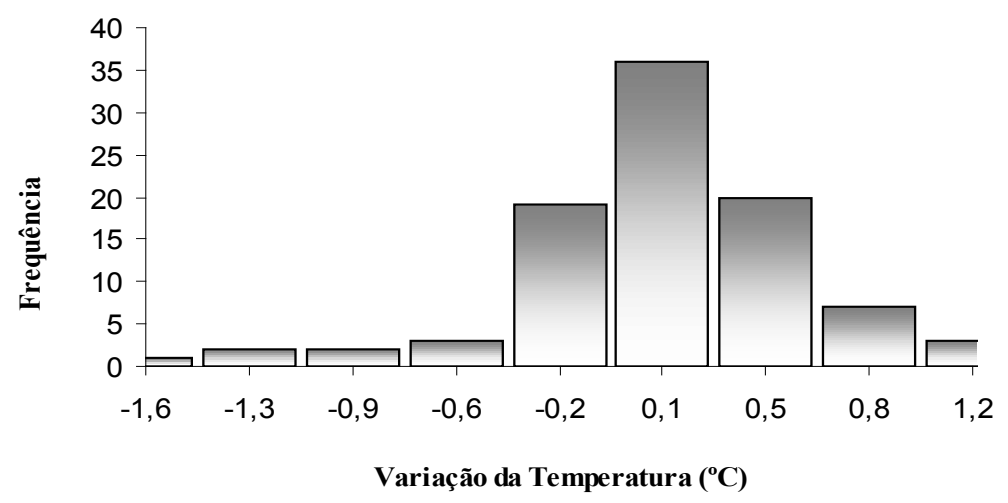

Figura 2. Variação $\left({ }^{\circ} \mathrm{C}\right)$ entre as Temperaturas médias mensais das máximas e das mínimas observadas e estimadas, para a região do Médio Paranapanema. 


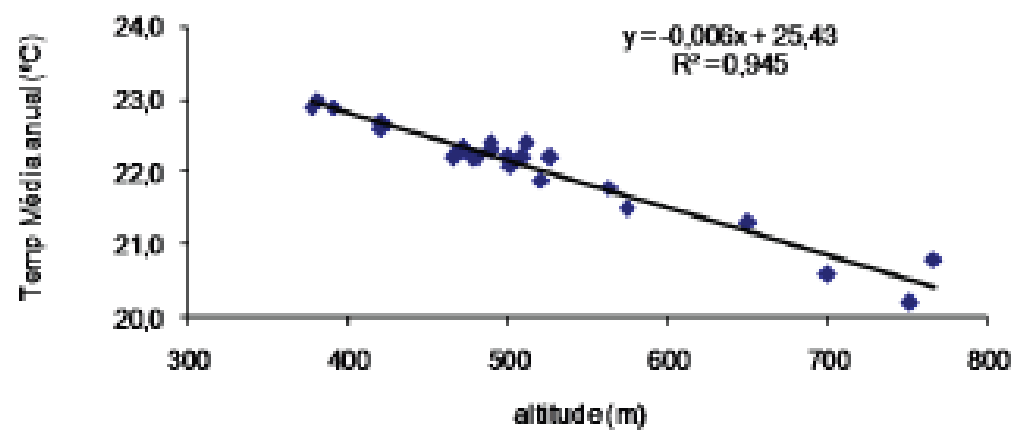

Figura 3. Relação entre altitude e valores estimados de temperatura média Anual para a região do Vale do Médio Paranapanema.

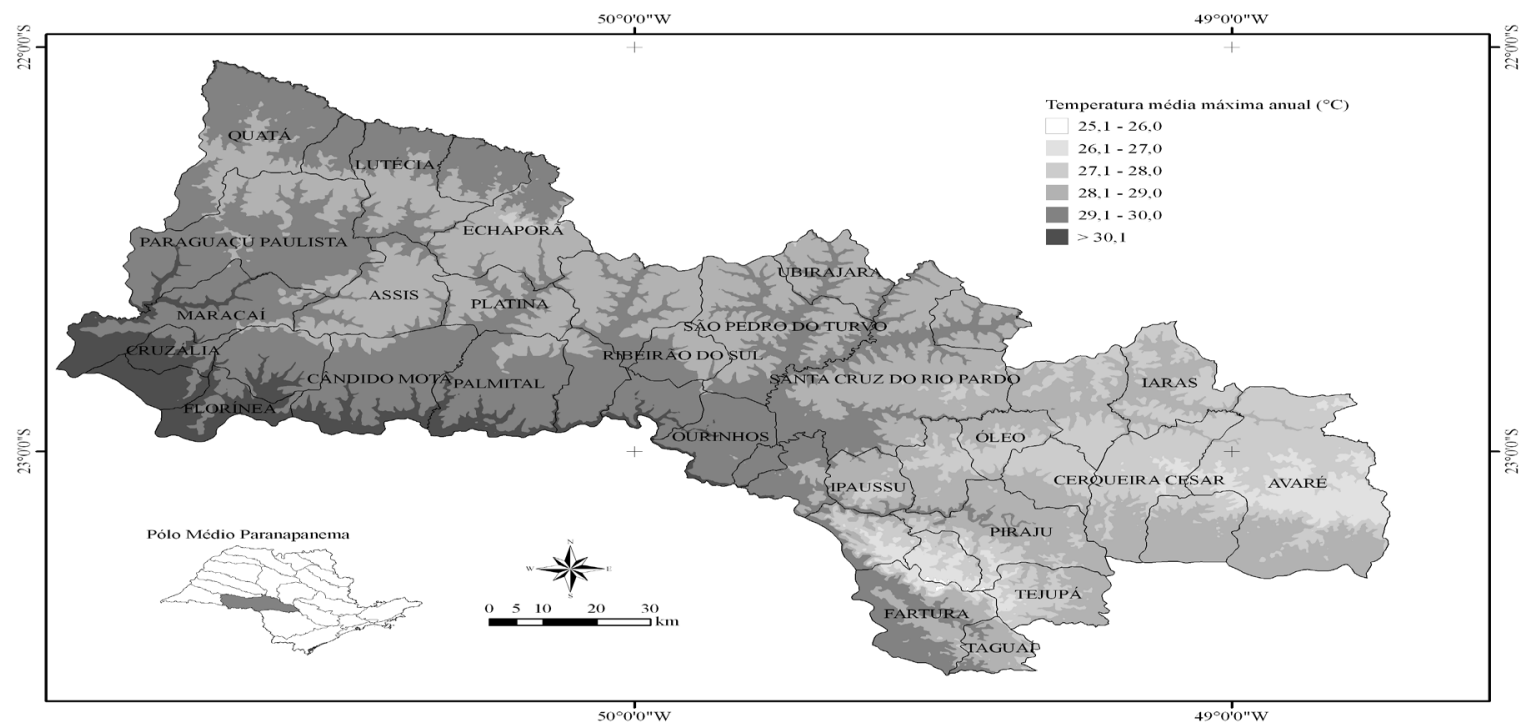

Figura 4. Temperatura média anual estimada das máximas para a região do Médio Paranapanema-SP.

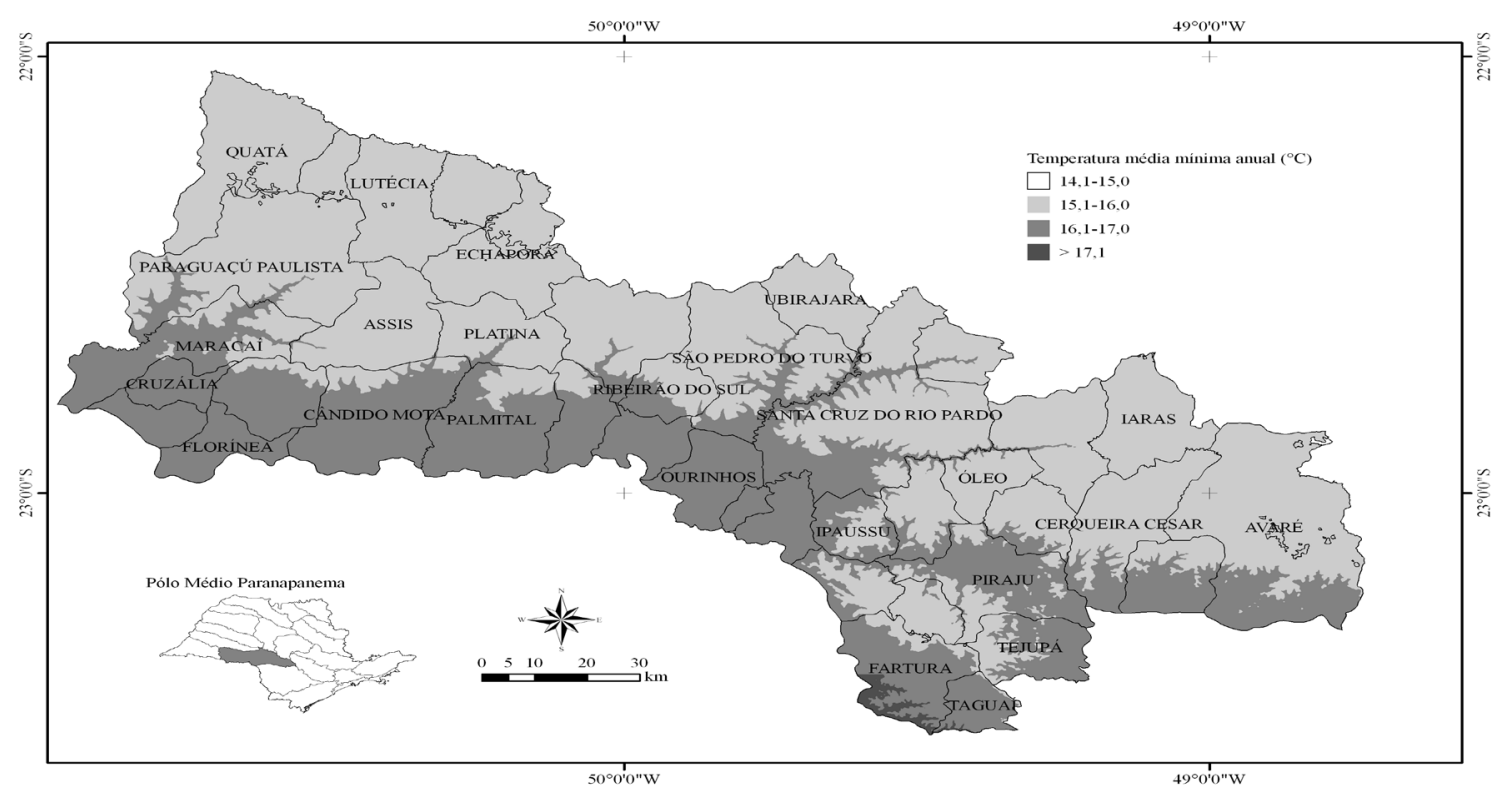

Figura 5. Temperatura média anual estimada das mínimas para a região do Médio Paranapanema, SP. 


\section{CONCLUSÕES}

- O método empregado para estimativa das temperaturas se mostrou adequado e com boa confiabilidade para estimar valores médios de temperaturas máximas e mínimas mensais para a região do Médio Paranapanema; e

- Encontrou-se alta relação para essa região entre altitude e médias anuais de temperatura. Assim, o modelo pode ser utilizado para estimativa de temperaturas médias anuais em todos os municípios da região do Médio Paranapanema.

As referências usadas são muito antigas e assumem que a temperatura do ar pode ser estimada em função apenas da influência individual de cada coordenada geográfica. Conseqüentemente, a previsibilidade desses modelos é muito baixa. Assim, os modelos de regressão obtidos apresentam uma previsibilidade muito baixa. Mais recentemente, estudos têm demonstrado que a temperatura do ar é uma função tanto das corrdenadas geográficas individualmente, mas também dos efeitos cruzados dessas coordenadas geográficas, conforme modelos proposro Silva et al. (2006):

$$
\begin{aligned}
& T_{i j}=a_{0}+a_{1} \lambda+\mathrm{a}_{2} \phi+\mathrm{a}_{3} \mathrm{~h}+\mathrm{a}_{4} \lambda^{2}+\mathrm{a}_{5} \phi^{2}+{ }_{6} h^{2} \\
& +\mathrm{a}_{6} h^{2}+\mathrm{a}_{7} \lambda \phi+\mathrm{a}_{8} \lambda \mathrm{h}+\mathrm{a}_{9} \phi \mathrm{h}+\mathrm{ASST}_{\mathrm{ij}}
\end{aligned}
$$

em que

$a_{0}, \ldots, \mathrm{a}_{9}=$ os coeficientes de regressão;

$1=$ longitude;

$\mathrm{f}=$ latitude; $\mathrm{e}$

$\mathrm{h}=$ elevação de cada posto analisada.

Os índices i e j indicam, respectivamente, o mês e o ano para o qual se está calculando a temperatura do ar $\left(T_{\mathrm{ij}}\right)$. Esses modelos melhoram sensivelmente a previsibilidade da estimativa da temperatura local e está disponível na página: www.dca.ufcg.edu.br.

\section{REFERÊNCIAS BIBLIOGRÁFICAS}

ABREU, P. G.; ABREU, V.M.V.; FRANCISCON,
L.; COLDEBEllA, A.; AMARAL, A.G. Estimativa da temperatura de globo negro a partir da temperatura de bulbo seco. Engenharia na Agricultura, Viçosa - MG, v.19, n.6, 2011.

ALFONSI, R.R.; PINTO, H.S.; PEDRO JÚNIOR, M.J. Estimativas das normais de temperaturas média mensal e anual do Estado de Goiás (BR) em função de altitude e latitude. Caderno de Ciências da Terra, São Paulo, v.45, n.1, p.1-6, 1974.

BARDIN, L.; MORAIS, J.F.L.; PEDRO JÚNIOR., M.J. Estimativa das temperaturas médias mensais das máximas e das mínimas para o Pólo Turístico do Circuito das Frutas, SP. Revista Brasileira de Engenharia Agrícola e Ambiental, Campina Grande, v. 14, n.6, p.618-624, 2010.

BURIOL, G.A.; ESTEFANEL, V.; FERREIRA, M.; PINTO, H.S. Estimativa das médias das temperaturas máximas mensais e anuais do Estado do Rio Grande do Sul. Revista do Centro de Ciências Rurais, Cascavel, v.3, n.1, p.131-150, 1973.

CAVALCANTI, E.P.; SILVA, E.D.V. Estimativa da temperatura do ar em função das coordenadas locais. In: CONGRESSO BRASILEIRO DE MALTEORXOLOGILA, 8. 1994, Belo Horizonte. Anais..., Belo Horizonte: Sociedade Brasileira de Meteorologia, 1994. v.1, p.154-157.

CIIAGRO - CENTRO INTEGRADO DE INFORMAÇÕES, AGROMETEOROLÓGICAS Disponível em <www.ciiagro.sp.gov.br> "Acesso em: 20 de nov $2010 "$ ".

FEITOZA, L.R. SCÁRDUA,J.A.; SEDIYAMA, G.C. et al. Estimativas das temperaturas médias das mínimas mensais e anual do Estado do Espírito Santo. Revista do Centro de Ciências Rurais, Santa Maria, v.9, n.3, p.279-291, 1979.

FEITOZA, L.R. SCÁRDUA,J.A.; SEDIYAMA, G.C. et al. Estimativas das temperaturas médias das máximas mensais e anual do Estado do Espírito Santo. Revista do Centro de Ciências Rurais, Santa Maria, v.10, n.1, p.15-24, 1980 a. 
FEITOZA, L.R. SCÁRDUA,J.A.; SEDIYAMA, G.C. et al. Estimativas das temperaturas médias das mínimas mensais e anual do Estado do Espírito Santo. Revista do Centro de Ciências Rurais, Santa Maria, v.10, n.1, p. 25-32, 1980 b.

FERNANDEZ, C.J. Simulation of normal annual and diurnal temperature oscillation in nonmountainous Mainland Unites States. Agronomy Journal, Madison, local, v.84, n.2, p.244-251, 1992.

FUMING, W. Method of temperature forescast for ten-days and its use in the mountainous region of west Hubei (China). Mountain Research, Beijing, v.6, n.1, p.38-41, 1988.

GALVANI, E.; KLOSOWSKI, E.S.; ESCOBEDO, J.F.; CUNHA, CUNHA, A.R. Modelo de estimativa de temperatura mínima do ar para a região de Maringá-PR. Revista Brasileira de Agrometeorologia, Santa Maria-Rs, v.8, n.1, p.105-110, 2000.

LADO, L.R.; SPAROVEK, G.; TORRADO, P.V.; DOURADO NETO, D. VAZQUEZ, F.M. Modelling air temperature for the satate of São Paulo, Brazil. Scientia Agrícola, Piracicaba, v.64, n.5, p.460-467, 2007.

LIMA, M.G.; RIBEIRO, V.Q. Equações de Estimativa da Temperatura do ar para o Estado do Piauí. Revista Brasileira de Agrometeorologia, Santa Maria, v.6, n.2, p.221-227, 1998.

MEDEIROS, S.S.; CECÍLIO, R.A.; MELO
JÚNIOR, J.C.F.; SILVA-JÚNIOR, J.L.C.Estimativa e espacialização das temperaturas mínima, média e máxima do território brasileiro situado entre $16 \mathrm{e}$ $24^{\circ}$ latitude sul e 48 e $60^{\circ}$ longitude oeste. Revista Brasileira de Engenharia Agrícola e Ambiental, Campina Grande, v.9, n.2, p.247-255, 2005.

MONTEIRO, C.A. de F. A dinâmica climática e as chuvas no estado de São Paulo: estudo geográfico em forma de atlas. São Paulo: IGEOG/ USP. 1973.

PEDRO JÚNIOR, M.J.; MELLO, M.H.A.; ORTOLANI, A.A.; ALFONSI, R.R.; SENTELHAS, P.C. Estimativa das temperaturas médias mensais das máximas e das mínimas para o Estado de São Paulo. Campinas, Instituto Agronômico, Boletim Técnico. 1991,11p.

PRELA, A. P.; RIBEIRO, A.M.A. Determinação de graus-dia acumulados e sua aplicação no planejamento do cultivo de feijão-vagem (Phaseolus vulgaris L.) para Londrina - PR. Revista Brasileira de Agrometeorologia, Santa Maria-RS, v.10, n.1, p.83-86, 2002.

PRELA-PANTANO, A.; ROLIM, G.S.; CAMARGO, M.B.P. de. Probabilidade de ocorrência de Temperaturas Mínimas menores que $5^{\circ} \mathrm{c}$ na região do Médio Paranapanema. Bragantia, Campinas, v.68, n.1, p.279-284, 2009.

SANDANIELO, A. Estimativa das temperaturas médias mensais e anuais na área do programa PÓLO NOROESTE PDRI-MT. Cuiabá: EMPAMT. 1987. 20 p. (Boletim de Pesquisa, 3). 